\title{
AN ADAPTIVE STEERABLE IMAGING ARRAY
}

\author{
E.K. HEGE and J.R.P. ANGEL \\ Steward Observatory, University of Arizona, Tucson AZ 85721, USA \\ and \\ A.D. GLECKLER, K.P. PFLIBSEN and B.L. ULICH \\ Kaman Aerospace Corporation, 3480 East Britannia, Tucson AZ 85706-5007, USA
}

January 26, 1993

\begin{abstract}
We propose an optimized design for the redeployment of the Multiple Mirror Telescope (MMT) primary optics as a $25 \mathrm{~m}$ Adaptive Steerable Imaging Array (ASIA) providing direct focal plane image formation. The design incorporates concepts of active telescope alignment and adaptive optics proven in the existing MMT. Basing predictions on contemporary radio telescope mount technology, $45 \mathrm{~m}$ to $100 \mathrm{~m}$ optical baselines may be practical.
\end{abstract}

Key words: Telescope design - High angular resolution - Adaptive optics

\section{Introduction}

The scientific and military communities continue to be interested in viewing space objects with increasing resolution. We propose an Adaptive Steerable Imaging Array (ASIA) which is a large, dilute-aperture, fully steerable, imaging telescope optimized for use at optical and infrared wavelengths. Adaptive optics, now being demonstrated with the present Multiple Mirror Telescope (Angel et al. 1990, Wizinowich et al. 1992, and Lloyd-Hart et al. 1993), is required for active optical metrology and inertial sensing to stabilize the optical segments against wind loading in a radio mount (Ulich et al. 1990a,b). ASIA does not depend upon aperture synthesis, which constructs an image from visibility measurements obtained from interferometry of the beams in various combinations. The ASIA design yields images directly in the focal plane of the combined beams just as the present MMT.

\section{A Redeployed MMT: Use Existing Resources and Technology}

As a result of the Steward Observatory borosilicate honeycomb mirror development program a seventh mirror may also be available, giving the light gathering capability of a $4.8 \mathrm{~m}$ aperture but with the resolution of a $25 \mathrm{~m}$ aperture, for a facility dedicated to the advancement of imaging technology and high angular resolution astronomy. The basic ASIA optical system, Table I, consists of seven cophased telescopes (1.8 $m$ diameter) arranged in a Cornwell (1988) circle. The seven apertures are centered on a $23 \mathrm{~m}$ circle at positions $0^{\circ}, \pm 53^{\circ}, \pm 98^{\circ}$, and $\pm 159^{\circ}$. This configuration yields an optimized coverage of the $u, v$-plane. The limiting resolution of the cophased system is a function of the diameter of the circle. The individual telescopes will be configured as long back-focal-distance Gregorian telescopes. An increased back focal length must be provided in order to accommodate the adaptive and beamcombining geometries for wide field-of-view imaging.

Existing 25 meter radio telescope mounts can be directly modified to provide support of the optics with sufficient optical rigidity against wind loading using 
off-axis stellar references. This is only feasible with comounted MMT-like imager designs which mitigate the field-of-view limitations of the long optical delay lines encountered in independent telescope aperture synthesis interferometric imager designs. The required technology has been refined by our team (Ulich, et al. 1990a,b) at the Multiple Mirror Telescope Observatory over the past decade and applied repeatedly elsewhere.

The use of a stellar reference for active optics alignment will provide the stable point spread function and cotemporaneous PSF determination necessary to calibrate and remove the effects of the large side-lobes inherent to the dilute pupil geometry. We propose using Multi-Conjugate Adaptive Optics (MCAO) in ASIA implemented with Kaman's PAMELA actuators (Gleckler et al. 1991) to provide a wide field of view with good correction in V supplementing ASIA's intrinsically diffraction-limited capability in infrared. The advantages expected from a twoconjugate MCAO system have recently been reviewed by Ellerbroek (1992).

The $u, v$-plane coverage and point-spread function expected for a $12 \mathrm{~m}$ redeployed MMT (Hege 1988) and wave-front sensed psf side-lobe self-calibration (Hege 1992) has been simulated. A dilute aperture $25 \mathrm{~m}$ ASIA would have angular resolution exceeding that of the largest existing direct imaging telescope (Keck 10 meter) by more than a factor of two, the largest existing dilute aperture telescope (the MMT) by a factor of three, and the Hubble Space Telescope by a factor of nine and with sensitivity similar to that ot the $5 \mathrm{~m}$ Hale telescope. A 25 meter diameter ASIA observing at $3.5 \mu \mathrm{m}$ will provide 30 milliarcsec (mas) resolution imagery $(\lambda / D)$ at its adaptive optics focus, without use of aperture synthesis techniques.

We avoid an expensive telescope enclosure, as well as costly, complex optical delay lines, in a cost effective installation, by providing weather proofing of the optics on its mount. A $25 \mathrm{~m}$ ASIA can be built in the near term with modest funding.

\section{An Extensible Configuration: The Design can Grow}

The ASIA concept has potential for extensibility to match, or possibly surpass, the high resolution and sensitivity capabilities of the proposed VLT and Keck arrays. With $502 \mathrm{~m}$ subapertures, figured as segments of a very fast (e.g. $\simeq f / 0.4$ ) off-axis paraboloid using Steward Observatory's stressed-lap polishing technique, a $100 \mathrm{~m}$ diameter ASIA would provide the light gathering capability of Keck + Keck2 with $\lambda / D=7$ mas at $3.5 \mu \mathrm{m}$. The $25 \mathrm{~m}$ ASIA would provide an extremely cost effective and unsurpassed dedicated astronomical optics laboratory for the design of such an international class $100 \mathrm{~m}$ facility, which at an appropriate Southern Hemisphere site would provide an outstanding facility for high angular resolution galactic astronomy in the Twenty-First Century.

\section{Acknowledgements}

EKH acknowledges the support of E.M. Hege and the University of Arizona Foundation for this work. 
TABLE I

Technical Characteristics of ASIA

\begin{tabular}{ll}
\hline Parameter & Value \\
\hline Maximum aperture (baseline) & $25 \mathrm{~m}$ \\
Number of subapertures & 7 \\
Subaperture diameter & $1.8 \mathrm{~m}$ \\
Pupil configuration & Cornwell ring \\
Sensitivity & $4.8 \mathrm{~m}$ \\
Strehl ratio & $>0.5$ \\
Adaptive optics: & \\
$\quad$ Low order (Gregorian) & Neural Net (Phase Diversity) \\
$\quad$ Higher order (Turbulence conjugate) & PAMELA (Shack-Hartmann) \\
\hline
\end{tabular}

TABLE II

Predicted Resolution of ASIA

\begin{tabular}{lllll}
\hline Parameter & Visible & \multicolumn{3}{l}{ Infrared } \\
\hline Wavelength $(\mu \mathrm{m})$ & 0.5 & \multicolumn{2}{l}{2.2} & \\
Array size & $25 \mathrm{~m}$ & $100 \mathrm{~m}$ & $25 \mathrm{~m}$ & $100 \mathrm{~m}$ \\
FWHM & & & & \\
$\quad$ (milliarcsec) & 4.7 & 1.2 & 21.0 & 5.2 \\
$\quad$ (nanoradian) & 23 & 5.8 & 100 & 25 \\
Spatial Resolution & & & & \\
$\quad$ LEO (m at 300km) & .007 & .00175 & .030 & .0075 \\
$\quad$ GEO (m at 60,000 km) & 1.4 & .35 & 6 & 1.5 \\
$\quad$ Solar system (km/AU) & 2.7 & .67 & 12 & 3 \\
$\quad$ Galactic (AU/kpc) & 4.1 & 1 & 18 & 4.5 \\
\hline
\end{tabular}

\section{References}

Angel, J.R.P., Wizinowich, P.L., Lloyd-Hart, M. and Sandler, D.: 1990, Nature 348, 221

Cornwell, T.: 1988, IEEE Trans. A. P. 36, 1165-1167

Ellerbroek, B.L.: 1992, in Real Time and Post-Facto Solar Image Correction, R.R. Radick, ed(s)., $13^{\text {th }}$ Sacramento Peak Summer Workshop, Sunspot, NM, In press

Gleckler, A.D. Markason, D.J. and Ames, G.H.: 1991, in Active and Adaptive Optical Components, M.A. Ealey, ed(s)., SPIE Proceedings 1542: Bellingham, WA, 176-189

Hege, E.K.: 1988, in Very Large Telescopes and their Instrumentation, M.-H. Ulrich, ed(s)., ESO: Garching, 805-814

Hege, E.K.: 1992, in High Resolution Imaging by Interferometry II, F. Merkle, ed(s)., ESO: Garching,

Lloyd-Hart, M., Dekany, R., McLeod, B., Wittman, D., Colucci, D., McCarthy, D. and Angel, R.: 1993, A strophys J. Letters 402, L81-L84

Ulich, B.L. and Rather, J.D.G.: 1990a, in Advanced Technology Optical Telescopes IV, L.D. Barr, ed(s)., SPIE Proceedings 1236: Bellingham, WA, 985-995

Ulich, B.L., Pflibsen, K., Sheppard, C. and Calmes, L.: 1990b, ibid , 768-776

Wizinowich, P., McLeod, B., Lloyd-Hart, M., Angel, J.R.P., Colucci, D., Dekany, R., McCarthy, D., Wittman, D. and Scott-Fleming, I.: 1992, A ppl. Opt. 31, 6036-6046 


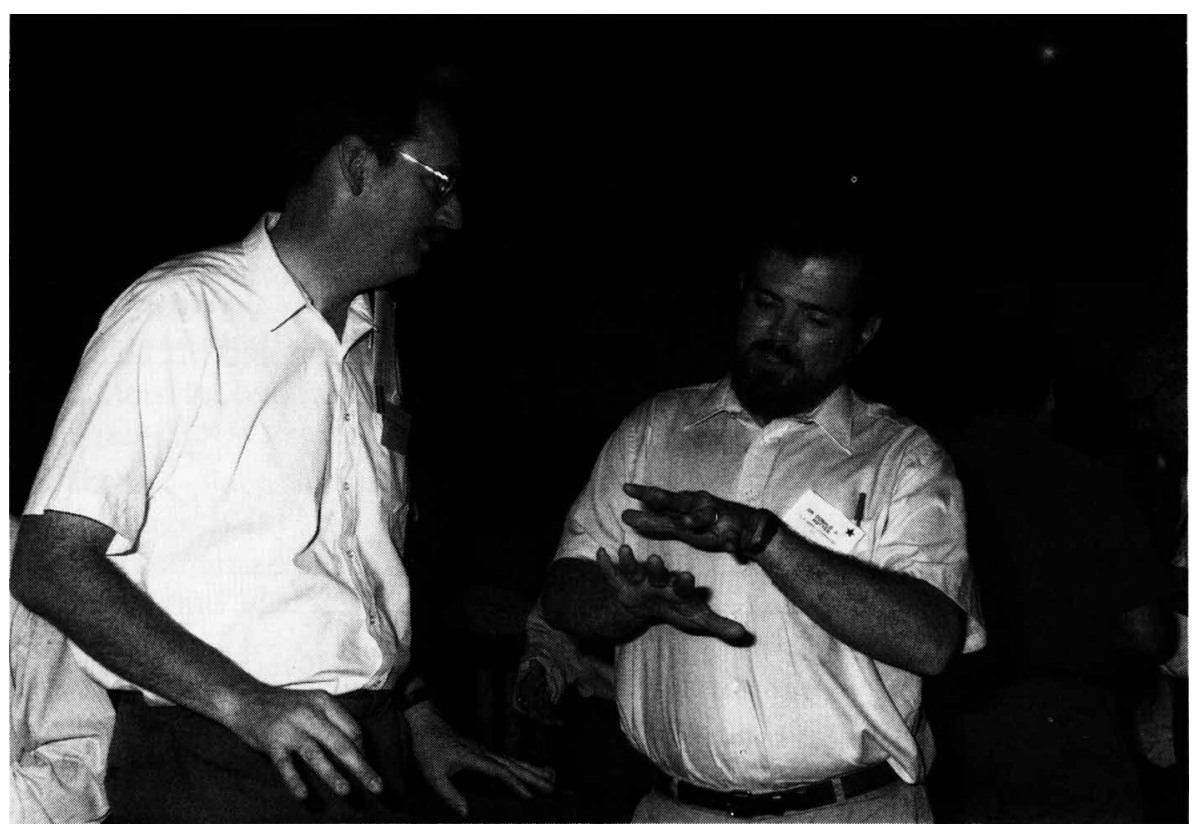

